\title{
Muerte súbita por embolia cerebral de mixoma de la aurícula izquierda
}

\section{Sudden death due to cerebral embolism from a left atrial myxoma}

\section{Resumen}

El mixoma es el tumor cardiaco primario más frecuente. Puede manifestarse con síntomas cardiacos, síntomas generales y embolismo tumoral sistémico. Aunque es un tumor benigno, las embolizaciones, especialmente las cerebrales, pueden ocasionar graves complicaciones neurológicas, de ahí que esté indicada su resección quirúrgica.

Presentamos el caso de un varón de 47 años que, en ausencia de síntomas previos, presenta síncope mientras mantenía relación sexual, falleciendo a las pocas horas. En la autopsia se constató en la aurícula izquierda, un tumor pediculado y gelatinoso de $1 \mathrm{~cm}$ de diámetro adherido al tabique interauricular, y en la arteria carótida interna derecha, un trombo oclusivo. Al estudio histológico, el tumor presentaba la morfología típica del mixoma cardiaco recubierto por trombo de fibrina y la oclusión arterial estaba ocasionada por émbolo tumoral y trombo de fibrina, hallazgos que explicaron el cuadro clínico.

Palabras clave: Muerte súbita. Tumor cardiaco. Mixoma. Embolia cerebral.

\section{Abstract}

Myxoma is the most common primary tumor of the heart. Clinical manifestations are due to cardiac disturbance, systemic embolism and constitutional symptoms. Although it is a benign tumor, embolism, especially cerebral ones, can produce neurological complications. Surgical treatment of the myxomas is indicated to prevent cerebral complications.

We present the case of a previously asymptomatic 47 years-old man who suffer syncope during sexual intercourse, dying few hours later without recover conscientious. At autopsy, in the left atria there was a gelatinous tumor, $1 \mathrm{~cm}$ length, attached to the interatrial septum and the internal right carotid artery was occluded by a thrombus. Microscopically, the tumor demonstrated the characteristic histological findings of cardiac myxoma covered by fibrinous thrombus and, the carotid artery was occluded by a tumoral embolism.

Key words: Sudden death. Cardiac tumour. Myxoma. Cerebral embolism.

\section{Introducción}

El mixoma es el tumor cardiaco primario más frecuente. Su histogénesis es incierta y ocurre exclusivamente en la superficie endocárdica. Generalmente es único y localizado en la aurícula izquierda, unido al tabique interauricular a nivel de la fosa oval. Aunque se ha descrito en un amplio espectro de edad, es más frecuente entre los 30-60 años y en mujeres. Es un tumor que puede manifestarse por una sintomatología y signos clínicos muy variables, simulando a veces enfermedades sistémicas, lo que dificulta y retrasa su diagnóstico. Las alteraciones clínicas que ocasionan los mixomas se han clasificado en tres tipos: síntomas cardiacos, síntomas generales o constitucionales que obedecen a un aumento del nivel sérico de interleukina-6, y síntomas secundarios a embolismos sistémicos, especialmente de tipo neurológico. La ecocardiografía transesofágica es el método idóneo para el diagnóstico clínico, pues los mixomas muestran una apariencia típica de masa móvil unida al endocardio, a veces pediculada.
B. Aguilera ${ }^{1}$

MP. Suárez-Mier ${ }^{1}$

R. García García ${ }^{2}$

${ }^{1}$ Doctora en

Medicina y Cirugía.

Especialista en

Anatomía Patológica.

Serv. de Histopatología del Instituto Nacional

de Toxicología y

Ciencias Forenses.

Departamento de

Madrid.

${ }^{2}$ Médico Forense. Instituto de Medicina Legal de Murcia.

Correspondencia: Dra. Beatriz Aguilera

Servicio de Histopatología INTCF-Madrid.

c/ José Echegaray 4. 28232 Las Rozas. Madrid. E-mail:

b.aguilera@mju.es

Fecha de recepción: 22.AG0.2011

Fecha de aceptación: 15.SEP.2011 
Aunque es un tumor histológicamente benigno, las embolizaciones, especialmente las neurológicas, pueden dejar graves secuelas, de ahí que esté indicada la resección quirúrgica ${ }^{1}$.
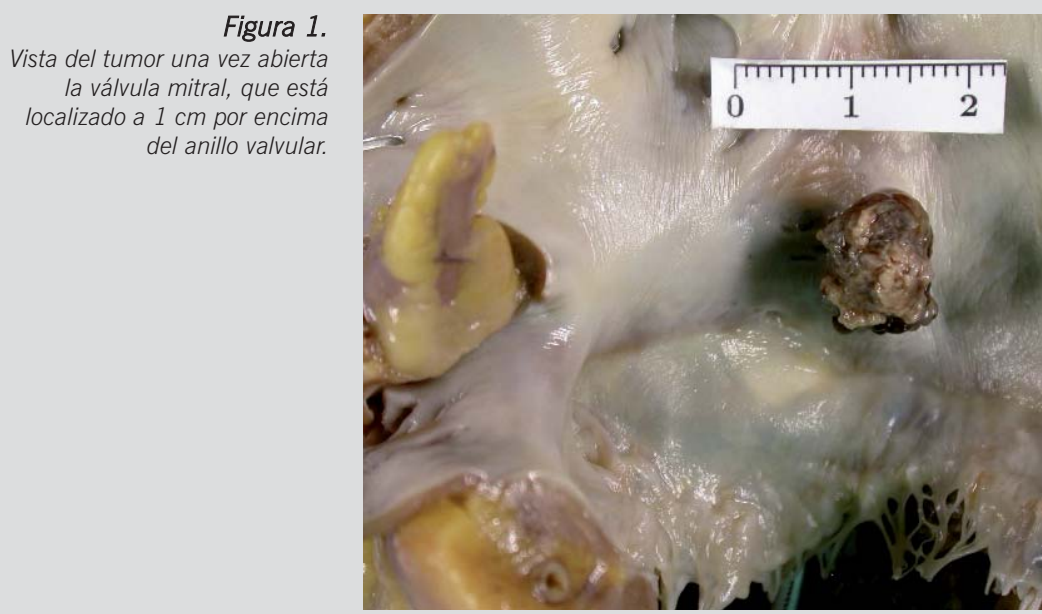

Figura 2. Aspecto histológico del tumor en el que se aprecia que está compuesto por un estroma laxo con células sueltas fusiformes o estrelladas o formando cordones que característicamente se disponen alrededor de los vasos (HE, 20x).

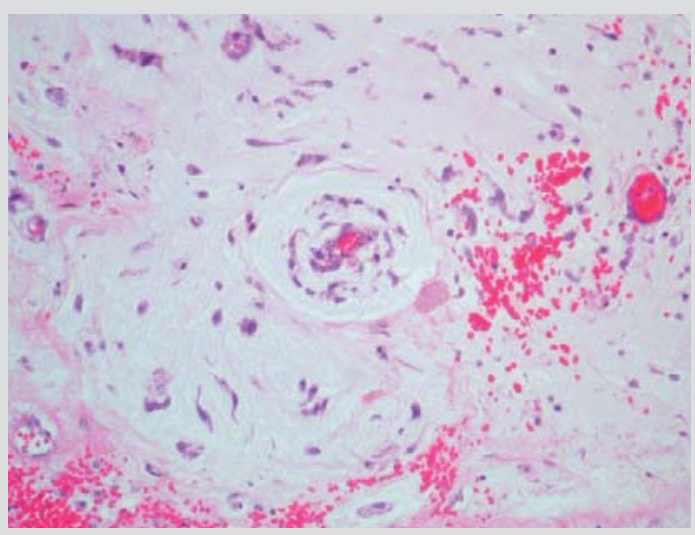

Figura 3.

El estroma laxo del mixoma se tiñe difusamente con el azul Alcian que demuestra su riqueza en mucopolisacáridos (tinción de PAS Azul Alcian, 10 x).

\section{Presentación del caso}

\section{Antecedentes}

Varón de 47 años, fumador, con antecedente de hábito enólico moderado y cólicos renales de repetición. Al parecer, tras disputa con su esposa, abandona el domicilio conyugal y horas más tarde, mientras mantenía relaciones sexuales con otra mujer, sufre episodio sincopal con pérdida de conciencia seguido de crisis tónico clónicas generalizadas y relajación de esfínteres. Se avisa al 061 que acude al domicilio, encontrándole inconsciente, Glasgow 3/15 con pupilas arreactivas, desviación de la mirada hacia la derecha y alteración del reflejo plantar. Le administran Midazolam y Norcurom, lo intuban y lo trasladan al hospital para su ingreso en UCl. Al ingreso, el TAC craneal simple fue normal, el Angio TAC demuestra falta de relleno de ambas ACMs y ACAs y ACP derecha y con el TAC perfusión hay falta de flujo al hemisferio derecho. La determinación de tóxicos en sangre y orina fue negativa. A las 14.30 horas, tras una nueva exploración neurológica y mediante EEG, se confirma el diagnóstico de muerte encefálica.

Tras dicho diagnóstico, se ponen en contacto con el médico forense de guardia solicitando autorización para la extracción de órganos para su trasplante, no concediéndose por no estar claras las circunstancias de su muerte: se maneja la posibilidad de posible compresión manual de estructuras cervicales como maniobra de excitación sexual mientras mantenía relaciones sexuales.

\section{Hallazgos de autopsia}

En el examen externo se apreciaron las lesiones propias del tratamiento médico hospitalario y las lesiones más importantes se encontraron en el corazón y el sistema nervioso. La disección cervical no reveló la existencia de lesión alguna ni en el hábito externo ni interno.

El corazón pesaba 432 g con hipertrofia concéntrica del VI. Los espesores de las paredes ventriculares son: VD $5 \mathrm{~mm}$, VI $15 \mathrm{~mm}$ y TIV $18 \mathrm{~mm}$. Los trayectos epicárdicos de las coronarias mostraban placas de ateroma calcificadas que ocasionaban una estenosis de hasta un $70 \%$ en la arteria coronaria descendente anterior y de un $80 \%$ en la coronaria circunfleja. En la aurícula izquierda, implantada en el tabique interauricular, a $1 \mathrm{~cm}$ por encima del anillo de la válvula mitral, existía una formación pediculada, 
de $1,2 \times 1 \mathrm{~cm}$, friable, rojiza, de aspecto gelatinoso y superficie irregular (Figura 1 ). Microscópicamente estaba constituida por células fusiformes sueltas 0 formando estructuras cordonales o anulares, algunas en disposición perivascular, inmersas en un estroma laxo que resultó positivo con la tinción de Azul Alcian. En su zona más superficial existían algunas células multinucleadas y áreas de hemorragia y el tumor estaba recubierto por trombo de fibrina con abundantes leucocitos polimorfonucleares (Figuras 2 y 3 ). No se encontraron émbolos tumorales en las arterias intramiocárdicas.

El encéfalo pesaba 1.590 g, las meninges estaban congestivas, sin hemorragias, y al examinar el polígono de Willis, se observó un trombo oclusivo en la arteria carótida interna derecha. Tras separar las estructuras de la fosa posterior se apreció hernia de uncus izquierdo y hemorragia en línea media del mesencéfalo (Figura 4). A los cortes coronales existía una zona de reblandecimiento y hemorragia en estructuras profundas del lado izquierdo próximas al tercer ventrículo, hemorragia en protuberancia y sangre en el cuarto ventrículo. Histológicamente, la luz de la arteria carótida interna derecha estaba ocluida por un émbolo tumoral semejante al de la aurícula izquierda y con fibrina condensada adherida (Figura 5). La amígdala cerebelosa izquierda presentaba numerosos focos de hemorragia perivascular y en una rama arterial un émbolo tumoral suelto en su luz.

\section{Diagnóstico anatomopatológico}

- Mixoma de la aurícula izquierda con trombo fibrino-leucocitario en superficie, y embolia tumoral en segmento terminal de la arteria carótida interna derecha y en ramas intraparenquimatosas del hemisferio izquierdo.

- Edema cerebral con signos de herniación transtentorial izquierda y hemorragia de Duret por enclavamiento.

- Ateroesclerosis coronaria calcificada con estenosis severa de la coronaria circunfleja.

- Hipertrofia concéntrica del ventrículo izquierdo.

\section{Discusión}

Los tumores cardiacos primarios son raros. Los mixomas son los más frecuentes, representando entre el $80-90 \%$ del total de los tumores según diferentes

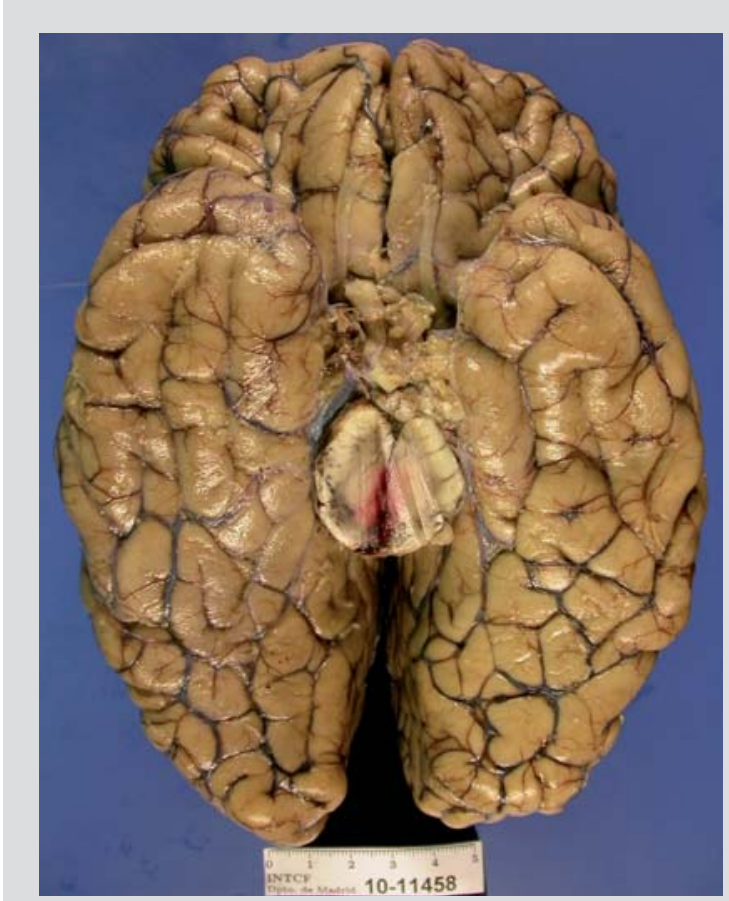

Figura 4.

Base del encéfalo.

Herniación del uncus izquierdo y hemorragia de Duret en tronco del encéfalo.

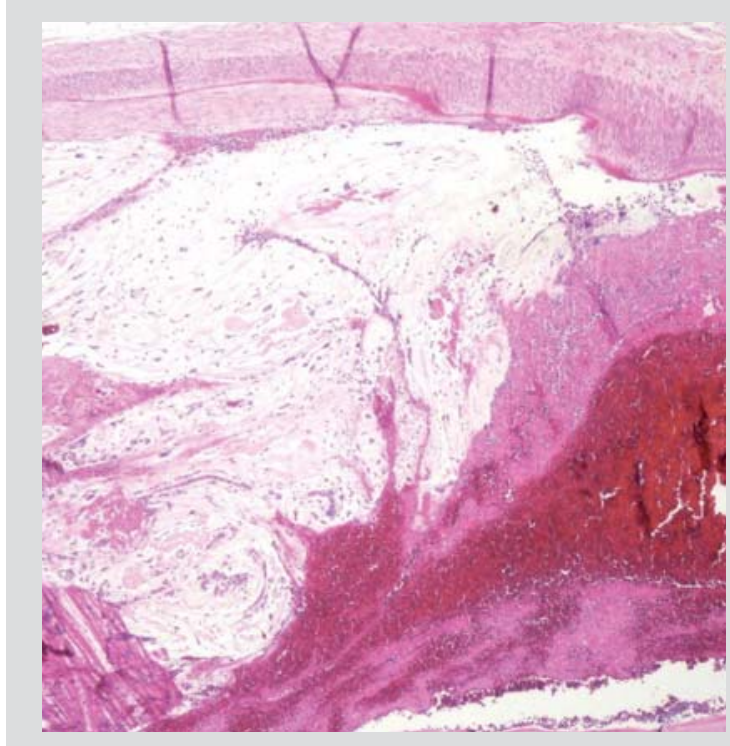

Figura 5.

Corte histológico de la arteria carótida interna derecha con su luz ocluida por émbolo de mixoma recubierto por trombo de fibrina (HE $4 \mathrm{x}$ ). series quirúrgicas ${ }^{2-5}$. Según Keeling et al, el tratamiento quirúrgico de los mixomas representa el $0,35 \%$ de las operaciones a corazón abierto ${ }^{6}$. El $90 \%$ se origina en la aurícula izquierda, la mayoría están implantados en el tabique interauricular a nivel de la fosa oval; con menor frecuencia se originan en la pared libre de la aurícula izquierda y excepcionalmente en la válvula mitral. La mayoría de los mixomas 
corresponden a la forma esporádica y son únicos. En menos de un $5 \%$ son familiares y forman parte del complejo de Carney descrito por este autor en los años 80. Este síndrome se hereda de forma autosómica dominante y se caracteriza por la presencia de lesiones cutáneas pigmentadas (nevos azules, lentiginosis), mixomas (cardíacos, cutáneos y mamarios), hiperreactividad endocrina hipersecretora, tumor de células de Sertoli del testículo y schwannomas melanóticos pigmentados. En este contexto los mixomas aparecen a edades más precoces, suelen ser múltiples y recidivan ${ }^{1,7}$.

Los mixomas pueden medir entre 1 y $13 \mathrm{~cm}$ de diámetro mayor y se han descrito dos formas macroscópicas: la forma sólida de superficie lisa y la forma polipoide o papilar gelatinosa con superficie verrucosa, pediculada y movible. En la correlación clínicopatológica, los primeros ocasionan con mayor frecuencia obstrucción y estenosis mitral con síntomas y signos de insuficiencia cardiaca y las formas pediculadas verrucosas producen con mayor frecuencia embolizaciones sistémicas y síntomas neurológicos. Ambos tipos puede ir acompañados de síntomas generales como pérdida de peso, astenia, febrícula y otros síntomas que simulan diversas enfermedades, y que desaparecen al resecar el tumor ${ }^{8}$.

El mixoma cardiaco puede debutar con clínica neurológica. En la recopilación de 107 casos publicados hecha por Ekinci y Donan ${ }^{9}$, la forma más frecuente de presentación fue un accidente cerebro-vascular isquémico en el $83 \%$ de los casos, con afectación de varios territorios en el $41 \%$. También puede manifestarse por síncope, cuadro psiquiátrico, cefalea y convulsiones $^{9}$. Son las formas papilares las que tienden a debutar con síntomas neurológicos ${ }^{4}$. En la serie de 26 mixomas de la aurícula izquierda publicada por Shimono et al, el 75\% de las formas papilares presentó infarto cerebral preoperatorio y solo el $12,5 \%$ de las formas sólidas ${ }^{8}$. En una serie de 28 mixomas estudiados en Barcelona, el 39,3\% de los afectados presentaron fenómenos embólicos pero solo el $21 \%$ debutaron con síntomas neurológicos. En 6/9 casos con síntomas neurológicos, se produjo un accidente isquémico transitorio que en 3 de ellos les ocasionó amaurosis. Los autores demuestran que afectan a personas más jóvenes y que el periodo desde que aparecen los síntomas hasta que se llega al diagnóstico de mixoma es en promedio de 2 años.
En este centro los mixomas cardiacos fueron el origen del $0,35 \%$ de los ACVA diagnosticados ${ }^{10}$. El clínico debe descartar un mixoma frente a un accidente isquémico cerebral en ausencia de otros factores de riesgo, especialmente si ocurre en adultos jóvenes ${ }^{11}$.

Los mixomas en un alto porcentaje tienen un trombo superficial que los recubre y que también puede embolizar. Los émbolos mixomatosos pueden invadir la pared de los vasos, debilitándola y favoreciendo la aparición de aneurismas que pueden dar síntomas años después de resecado el mixoma cardiaco. Excepcionalmente se han descrito metástasis de mixoma en el cerebro que se manifiestan por efecto masa $^{12,13}$

Se han descrito casos con clínica de obstrucción arterial periférica, en que el estudio histológico del émbolo resecado ha permitido llegar al diagnóstico de mixoma cardiaco ${ }^{14}$. Excepcionalmente los mixomas pueden infectarse y dar una clínica que semeja una endocarditis infecciosa ${ }^{15,16}$. En los últimos años y basado en el hallazgo histopatológico de que muchos mixomas cardiacos se asocian a un trombo superficial, se han tratado los émbolos mediante trombolisis para recuperar la permeabilidad vascular ${ }^{17,18}$.

Se ha estimado que entre el $8-10 \%$ de los mixomas cardiacos son asintomáticos ${ }^{3,4}$. En los 83 casos de mixomas de la aurícula izquierda estudiados en el Instituto de Patología de las Fuerzas Armadas (AFIP, Washington) en el $19 \%$ fue un hallazgo incidental ${ }^{19}$. Probablemente esta diferencia se deba al tipo de población estudiada. El caso que presentamos era al parecer asintomático hasta que presenta el episodio agudo que le lleva al fallecimiento.

Los mixomas de la aurícula izquierda excepcionalmente producen muerte súbita. Ocurre en el $4 \%$ en la serie de la AFIP ${ }^{19}$. En la recopilación de los 17 casos publicados hecha por Modi et a/20, la muerte súbita ocurre con mayor frecuencia en varones y tras una sintomatología clínica que ha variado entre 1 día y varios meses. Puede ocurrir a cualquiera edad y es excepcional que debute con un síncope como en nuestro caso y en contados casos publicados ${ }^{21}$. También se ha descrito muerte súbita ocasionada por embolismo pulmonar de un mixoma originado en la aurícula derecha ${ }^{22}$. 


\section{Bibliografía}

1. Burke A, Jeudy J, Virmani R. Cardiac Tumors: an update. Heart. 2008;94:117-23.

2. Fernandes F, Soufen HN, lanni BM, et al. Primary Neoplasms of the Heart. Clinical and Histological Presentation of 50 Cases. Arq Bras Cardiol. 2001;76:231-7.

3. Pinede $L$, Duhaut $P$, Loire R. Clinical presentation of left atrial cardiac myxoma. A series of 112 consecutive cases. Medicine (Baltimore). 2001;80:159-72.

4. Swartz M, Lutz C, Chandan V, et al. Atrial Myxomas: Pathologic Types, Tumor Location, and Presenting Symptoms. J Card Surg. 2006;21:435-40.

5. García Zubiri C, Yebra Yebra M, Salas Natón C, et al. Mixoma cardiaco: serie de 30 pacientes. Rev Clin Esp. 2009;209:478-82.

6. Keeling I, Oberwalder P, Anelli-Monti M, et al. Cardiac myxomas: 24 years of experience in 49 patients. Eur J Cardio-Thoracic Surg. 2002;22:971-7.

7. Carney JA. Carney complex: the complex of myxomas, spotty pigmentation, endocrine overactivity, and schwannomas. Semin Dermatol. 1995;14:90-8.

8. Shimono T, Makino S, Kanamori Y. Left atrial myxomas. Using Gross Anatomic Tumor Types to Determine Clinical Features and Coronary Angiographic Findings. Chest. 1995;107:674-9.

9. Ekinci EL, Donnan GA. Neurological manifestations of cardiac myxoma: a review of the literature and report of cases. Intern Med J. 2004;34:243-9.

10. Álvarez-Sabín J, Lozano M, Sastre-Garriga J, et al. Transient Ischemic Attack: A common initial manifestations of Cardiac Myxoma. Eur Neurol. 2001;45:165-70.

11. Yeh HH, Yang CC, Tung WF, et al. Young stroke, cardiac myxoma, and multiple emboli: a case report and literature review. Acta Neurol Taiwan. 2006; 15:201-5.
12. Lee V, Connolly H, Brown R Jr. Central nervous system manifestations of cardiac myxoma. Arch Neurol. 2007;64:1115-20.

13. Herbst M, Wattjes M, Urbach $\mathrm{H}$, et al. Cerebral Embolism from Left Atrial Myxoma Leading to Cerebral and Retinal Aneurysms: A Case Report. Am J Neuroradiology. 2005;26:666-9.

14. Val-Bernal JF, Acebo E, Gómez-Roman JJ, et al. Anticipated diagnosis of left atrial myxoma following histological investigation of limb embolectomy specimens: A report of two cases. Pathol Int. 2003;3:489-94.

15. García-Quintana A, Martín-Lorenzo P, Suárez de Leso $\mathrm{J}$, et al. Infected Left Atrial Myxoma. Rev Esp Cardiol. 2005;58:1358-60.

16. Janion M, Sielski J, Ciuraszkiewickz K. Sepsis complicating giant cardiac myxoma. Am J Emerg Med. 2008;26:387;e.3-4.

17. Ibrahim M. Biatrial Myxoma and Cerebral Ischemia. Successfully Treated with Intravenous Thrombolytic Therapy and Surgical Resection. Tex Heart Inst J. 2008;35:193-5.

18. Mu-Chien Sun, Hui-Chun Tai, Chien-Hui Lee. Intravenous Thrombolysis for Embolic Stroke due to Cardiac Myxoma. Case Rep Neurol. 2011;3:21-26.

19. Burke A, Virmani R. Cardiac Myxoma en Tumors of the Heart and Great Vessels. Washington: Armed Forces Institute Pathology, 1996;pp. 21-46.

20. Modi K, Venkatesh P, Agnani S, et al. Sudden death in a Patient with Left Myxoma: Report of two cases and review of the literature. BJMP 2010;3(2):318.

21. Akyıldız EÜ, Tolgay E, Öz B, et al. Cardiac myxoma: an unusual cause of sudden death in childhood. Turk J Pediatr. 2006;48:172-4.

22. Fracasso T, Varchmin-Schultheiss $K$. Sudden death due to pulmonary embolism from right atrial myxoma. Int J Legal Med. 2009;123:157-9. 